\title{
MODELLING A LARGE-SCALE UNIAXIAL SHAKING TABLE FACILITY
}

\author{
Paola Ceresa $^{1}$, Franco Brezzi ${ }^{1}$ and Gian Michele Calvi ${ }^{1}$ \\ ${ }^{1}$ Istituto Universitario di Studi Superiori di Pavia (IUSS) \\ Piazza della Vittoria 15, 27100 Pavia (Italy) \\ paola.ceresa@iusspavia.it, franco.brezzi@iusspavia.it,gm.calvi@iusspavia.it
}

Keywords: Large-scale dynamic testing facility, nonlinear kinematics, high-order nonlinear differential equations, numerical solver, experimental data

\begin{abstract}
Aim of this research is the development and the calibration of a mathematical model for simulating the response of a large-scale uniaxial shaking table facility. The system to be modelled is composed of several interacting components such as the shaking table, the specimen to be tested and the reaction mass. The latter could be isolated by air suspension springs and damped by heavy duly automotive shock absorbers or could rest directly on the supporting soil. The proposed model has been developed to take into account the stiffness and the damping due to suspension system/shock absorber system as well as the flexibility of the supporting soil.
\end{abstract}

The main novelty of the proposed model is the nonlinear kinematics taken into account in the formulation of the equations of motion of the dynamic system. The rocking of the reaction mass is modelled and the nonlinear kinematics due to large rotations associated to an intended uniaxial motion of the shaking table is considered.

The nonlinear equations of motions are derived using the Lagrangian approach under the assumption of large displacements and large rotations.

The proposed mathematical model has been derived to be general and capable of predicting the response of any given uniaxial shaking table whose response could be strongly affected by the rocking of the reaction mass.

The derived system of nonlinear equations of motion has been applied for modelling the response of an existing large-scale dynamic testing facility - the shaking table of the Eucentre Laboratory. The obtained results seem very promising, useful for understanding the causes of the complex behaviour of the dynamic testing facility and instrumental for helping in future experimental campaigns. 


\section{INTRODUCTION}

Large servo-hydraulic shaking table systems are essential tools in experimental earthquake engineering since they provide effective ways to subject structures to dynamic excitations similar to those induced by real earthquakes. Several shaking tables throughout Europe are being used to investigate the dynamic effects of earthquake on structures. The latter are usually characterised by more than one degree of freedom ( 3 and 6 DOFs are common), a payload of about 20-30 tonnes and limited performances in terms of payload, peak velocities, peak accelerations and stroke. With regards to the initiatives outside Europe, considerable funding and effort have been allotted over the past 40 years in the construction of continuously larger and powerful shaking table facilities [1]. Thus, the need for building large and powerful dynamic facilities capable of testing up to failure full-scale models has become clear.

In the search of improved earthquake simulation, increasing accuracy with which a shaking table can replicate a desired motion is of great interest. A comprehensive understanding of the dynamic characteristics of a shaking table is of fundamental importance for optimising its control algorithm and, eventually, assessing its limitations. Therefore, a mathematical model of the complete shaking table system is required for planning future experiments, optimising the system, understanding the causes of a complex behaviour and developing safety measurements.

The current study is devoted to a large-scale dynamic testing facility composed by the specimen (i.e., payload), shaking table, reaction mass, airbags, dampers and soil. In particular, the subject of the research is a large-scale and powerful shaking table designed for performing single-axis tests (1 DOF) whose behaviour could be affected by the rocking of the reaction mass. The effects of the support system (i.e. reaction mass, airbags, dampers and soil) interaction on the response of the shaking table are evaluated developing a deterministic model capable to take into account horizontal, vertical and rotational accelerations of the overall dynamic testing facility.

\section{DERIVATION OF THE DETERMINISTIC MODEL}

The behaviour of a large and powerful dynamic testing facility could be characterised and influenced by: i) large overturning moment due to the specimen located on the shaking table; ii) large lateral displacement of the shaking table as well as powerful performances in terms of both velocity and acceleration; iii) unbalanced configuration of the reaction mass; iv) deformability of the soil. All these factors could cause the presence of non-negligible nonlinear terms in the equations of motion describing the dynamic testing facility.

The model to be derived must be as general as possible and applicable to any given shaking table. The dynamics of the shaking table cannot be described by a single equation of motion related to its allowed longitudinal translation since the interactions between the specimen, shaking table, reaction mass, isolation and damping systems, foundation and soil have to be taken into account. The derived model has to be capable of simulating the behaviour of a shaking table that could be subjected to a possible large rotation since it moves in a rotating frame of reference which corresponds to the support system. Therefore, the motion of the shaking table has to be described by a set of equations written considering a reference system characterised by longitudinal and vertical translations and rotational degrees of freedom. The out-of-plane motion is not accounted for in this phase of the formulation. The Lagrange's approach for deriving the nonlinear equations of motions under the assumptions of large displacements is applied. In addition, the homogeneous matrix approach proposed by Legnani et al. $[2,3]$ is adopted since it allows to study kinematic and dynamic motions of rigid bodies in the form of a consistent method employing $4 \times 4$ matrices, following Lagrange's method. A 
comprehensive description of the derivations of equations is given in [4]. However, a brief summary of the main assumptions and findings is given in the following sections in order to clearly catch the novelty of the proposed deterministic model and best exploit the potentials of the proposed model for studying complex dynamic problems as the validation examples discussed in Section 4.

Figure 1 shows the two main systems are modelled. The first (later named system $I$ ) is composed by the reaction mass, the isolators, the dampers and the soil. The second (system II) includes the shaking table and the payload to be tested. Three independent coordinates are introduced to describe the horizontal and vertical displacements and the rotation of the system I $-\mathrm{Q}_{1}, \mathrm{Q}_{2}$ and $\mathrm{Q}_{3}$, respectively. The horizontal displacement between system II and system I is the fourth generalised coordinate $-\mathrm{Q}_{4}$. It is assumed that the payload is perfectly restrained to the platform (of the shaking table) and it moves accordingly to the shaking table motion. Therefore, the relative vertical displacement $\mathrm{Q}_{5}$ and the relative rotation $\mathrm{Q}_{6}$ are disregarded and the rotation $\mathrm{Q}_{3}$ becomes the rotation of both system I and system II. Hence, four differential equations are derived with respect to the coordinates $\mathrm{Q}_{1}, \mathrm{Q}_{2}, \mathrm{Q}_{3}$ and $\mathrm{Q}_{4}$. According to the homogeneous matrix approach [2,3], the ingredients for writing the Lagrange's equation of motion are the position, velocity and acceleration matrices $-\mathbf{M}, \mathbf{W}$ and $\mathbf{H}$, respectively which allow to handle both linear and angular terms at the same time. In addition, the pseudoinertial matrix (J) which describes the mass distribution of a body has to be introduced. The relative motion between system I and system II is described using familiar concepts as the velocity composition theorem (or Rival's theorem) and Coriolis' theorem. Three frames of reference are considered during the derivation of the deterministic model: $\left(X_{1}, Y_{1}, Z_{1}\right)$ is the absolute inertial frame of reference; $\left(X_{2}, Y_{2}, Z_{2}\right)$ and $\left(X_{3}, Y_{3}, Z_{3}\right)$ are embedded in the centres of gravity of system $I$ and system II, respectively.

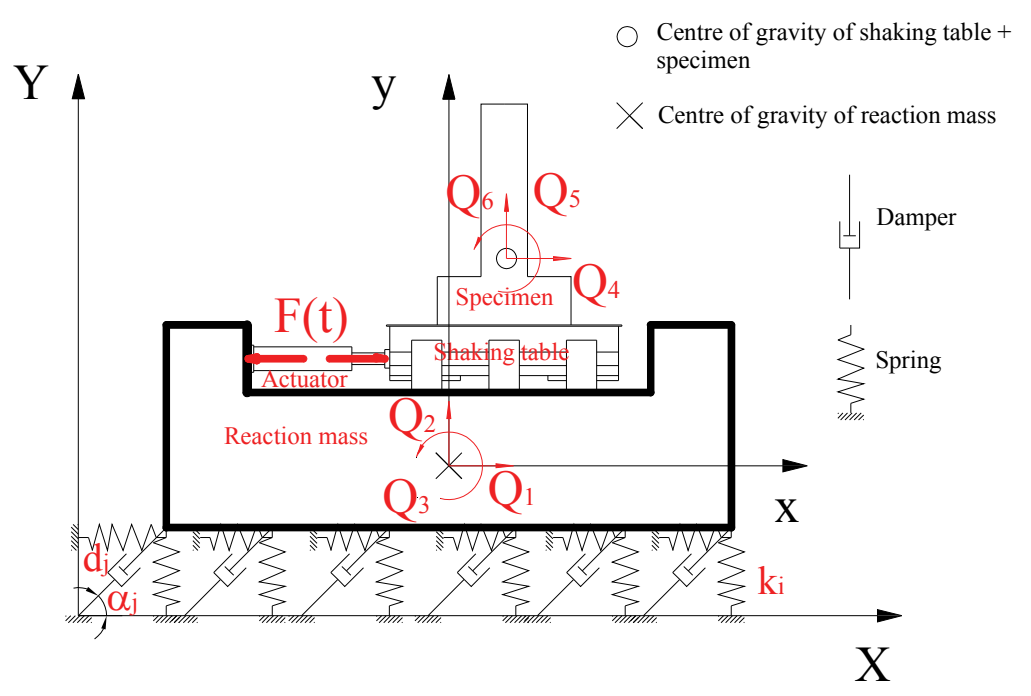

Figure 1: Dynamic testing facility to be modelled.

\subsection{Kinetic energy of the overall dynamic system}

The total kinetic energy $(T)$ is the sum of the kinetic energies of system I and system II:

$$
\begin{aligned}
& T_{I}=(1 / 2) \cdot \operatorname{Trace}\left(\mathbf{W}_{12(1)} \mathbf{J}_{\mathrm{I}(2)} \mathbf{W}_{12(1)}{ }^{T}\right) \\
& T_{I I}=(1 / 2) \cdot \operatorname{Trace}\left(\mathbf{W}_{13(1)} \mathbf{J}_{\mathrm{II}(1)} \mathbf{W}_{13(1)}{ }^{T}\right)
\end{aligned}
$$


where $\mathbf{J}_{I(2)}$ is the pseudo-inertial matrix of system $I$ in the reference frame $\left(X_{2}, Y_{2}, Z_{2}\right), \mathbf{J}_{I I(1)}$ is the pseudo-inertial matrix of system II in the reference frame $\left(\mathrm{X}_{1}, \mathrm{Y}_{1}, \mathrm{Z}_{1}\right), \mathbf{W}_{12(1)}$ and $\mathbf{W}_{13(1)}$ are the velocity matrices of system I and system II, respectively. The matrices that characterise the motion of system $I$ are given in Eq. (2), where $m_{I}$ is the mass of the system:

$$
\begin{gathered}
\mathbf{M}_{12(1)}=\left[\begin{array}{cccc}
\cos Q_{3} & -\sin Q_{3} & 0 & Q_{1} \\
\sin Q_{3} & \cos Q_{3} & 0 & Q_{2} \\
0 & 0 & 1 & 0 \\
0 & 0 & 0 & 1
\end{array}\right], \mathbf{W}_{12(1)}=\left[\begin{array}{cccc}
0 & -\dot{Q}_{3} & 0 & \dot{Q}_{1} \\
\dot{Q}_{3} & 0 & 0 & \dot{Q}_{2} \\
0 & 0 & 0 & 0 \\
0 & 0 & 0 & 0
\end{array}\right] \\
\mathbf{J}_{\mathrm{I}(2)}=\left[\begin{array}{cccc}
I_{x x, I} & 0 & 0 & 0 \\
0 & I_{y y, I} & 0 & 0 \\
0 & 0 & I_{z z, I} & 0 \\
0 & 0 & 0 & m_{I}
\end{array}\right]=\left[\begin{array}{cccc}
\int x^{2} d m_{I} & 0 & 0 & 0 \\
0 & \int y^{2} d m_{I} & 0 & 0 \\
0 & 0 & \int z^{2} d m_{I} & 0 \\
0 & 0 & 0 & m_{I}
\end{array}\right]
\end{gathered}
$$

The velocity matrix $\mathbf{W}_{13(1)}$ of the system $I I$ with respect to $\left(\mathrm{X}_{1}, \mathrm{Y}_{1}, \mathrm{Z}_{1}\right)$ is given by the sum of the drag component $\mathbf{W}_{12(1)}$ and the relative velocity $\mathbf{W}_{23(1)}$, according to the velocity composition theorem:

$$
\mathbf{W}_{13(1)}=\mathbf{W}_{12(1)}+\mathbf{W}_{23(1)}=\mathbf{W}_{12(1)}+\mathbf{M}_{12(1)} \cdot \mathbf{W}_{23(2)} \cdot \mathbf{M}_{21(1)}
$$

The position and velocity matrixes of the system II in the frame of reference $\left(\mathrm{X}_{2}, \mathrm{Y}_{2}, \mathrm{Z}_{2}\right)$ are equal to:

$$
\mathbf{M}_{23(2)}=\left[\begin{array}{cccc}
1 & 0 & 0 & Q_{4} \\
0 & 1 & 0 & 0 \\
0 & 0 & 1 & 0 \\
0 & 0 & 0 & 1
\end{array}\right], \quad \mathbf{W}_{23(2)}=\left[\begin{array}{cccc}
0 & 0 & 0 & \dot{Q}_{4} \\
0 & 0 & 0 & 0 \\
0 & 0 & 0 & 0 \\
0 & 0 & 0 & 0
\end{array}\right]
$$

Using the general transformation law that works for pseudo-tensor of inertia [3], the mass distribution of the system II in $\left(\mathrm{X}_{1}, \mathrm{Y}_{1}, \mathrm{Z}_{1}\right)$ can be computed according to this expression:

$$
\mathbf{J}_{\mathrm{II}(1)}=\mathbf{M}_{12(1)}\left(\mathbf{M}_{23(2)} \mathbf{J}_{\mathrm{II}(3)} \mathbf{M}_{23(2)}^{T}\right) \mathbf{M}_{12(1)}^{T}
$$

where $\mathbf{J}_{\mathrm{II}(3)}$ is the pseudo-matrix of inertia of system $I I$ when the frame of reference $\left(\mathrm{X}_{3}, \mathrm{Y}_{3}\right.$, $\left.Z_{3}\right)$ is concerned.

$$
\mathbf{J}_{I I(3)}=\left[\begin{array}{cccc}
I_{x x, I I} & 0 & 0 & 0 \\
0 & I_{y y, I I} & 0 & 0 \\
0 & 0 & I_{z z, I I} & 0 \\
0 & 0 & 0 & m_{I I}
\end{array}\right]=\left[\begin{array}{ccccc}
\int x^{2} d m_{I I} & 0 & 0 & 0 \\
0 & \int y^{2} d m_{I I} & 0 & 0 \\
0 & 0 & \int z^{2} d m_{I I} & 0 \\
0 & 0 & 0 & m_{I I}
\end{array}\right]
$$

The final expression of $T$ is given by: 


$$
T=\left\{\begin{array}{l}
0.5 m_{I}\left[\dot{Q}_{1}^{2}+\dot{Q}_{2}^{2}\right]+(1 / 2)\left(I_{x x, I}+I_{y y, I}\right) \dot{Q}_{3}^{2}+ \\
+0.5 m_{I I}\left[\dot{Q}_{1}^{2}+\dot{Q}_{2}^{2}+\dot{Q}_{4}^{2}+2 \dot{Q}_{1} \dot{Q}_{4} \cos Q_{3}+2 \dot{Q}_{2} \dot{Q}_{4} \sin Q_{3}\right]+ \\
0.5 \dot{Q}_{3}^{2}\left[\left(I_{x x, I I}+I_{y y, I I}\right)+m_{I I}\left(Q_{1}^{2}+Q_{2}^{2}+Q_{4}^{2}+2 Q_{1} Q_{4} \cos Q_{3}+2 Q_{2} Q_{4} \sin Q_{3}\right)\right]+ \\
+m_{I I} \dot{Q}_{3}\left[Q_{1} \dot{Q}_{2}-Q_{2} \dot{Q}_{1}+\left(Q_{1} \dot{Q}_{4}-Q_{4} \dot{Q}_{1}\right) \sin Q_{3}+\left(-Q_{2} \dot{Q}_{4}+Q_{4} \dot{Q}_{2}\right) \cos Q_{3}\right]
\end{array}\right.
$$

\subsection{Potential energy of the overall dynamic system}

The total potential energy $V$ of the system is computed as the sum of the contributions due to gravity (Eq. 8), the $n s$ springs $k_{\mathrm{i}}$ introduced to model the isolators and the stiffness of the soil (whose slopes could be general, different from each other and represented by the angle $\alpha_{\mathrm{i}}$ ) as shown in Eq. (9) and the translational spring $k_{4}$ representing the stiffness of the servohydraulic actuator, Eq. (10):

$$
\begin{gathered}
\mathrm{V}_{P i}=\operatorname{Trace}\left(-\mathbf{H}_{g} \cdot \mathbf{J}_{i}\right) \text { for } \mathrm{i}=I, I I \\
V_{i_{\text {_springs }}}=0.5 \sum_{i=1}^{n s} k_{i} \bar{u}_{i}^{2}=0.5 \sum_{i=1}^{n s} k_{i}\left\{\begin{array}{l}
{\left[\left(\cos Q_{3}-1\right) X_{i}-\sin Q_{3} Y_{i}+Q_{1}\right]\left(\cos \alpha_{i}\right)+{ }^{2}} \\
\left.+\sin Q_{3} X_{i}+\left(\cos Q_{3}-1\right) Y_{i}+Q_{2}\right]\left(\sin \alpha_{i}\right)
\end{array}\right\}^{2} \\
V_{k 4}=0.5 k_{4}\left(U_{2}-U_{1}\right)^{2} 0.5 k_{4}\left[Q_{4}+\bar{x} Q_{3} \sin Q_{3}-\bar{y} Q_{3} \cos Q_{3}\right]^{2}
\end{gathered}
$$

where $\mathbf{H}_{\mathrm{g}}$ is the matrix of gravity acceleration (whose only non-null component is in the $\mathrm{y}$ direction, $\left.g_{\mathrm{y}}\right), \bar{u}_{i}$ is the elongation or shortening of the $k_{\mathrm{i}}$ springs, $X_{\mathrm{i}}$ and $Y_{\mathrm{i}}$ are the coordinates of the $i$-th spring, $U_{1}$ and $U_{2}$ are the displacements of the two ends of the actuator computed in a rotated frame of reference. The total potential energy $V$ is obtained as:

$$
\begin{gathered}
V=V_{P I}+V_{P I I}+V_{i_{-} \text {springs }}+V_{k 4} \\
V=g_{y} m_{I} Q_{2}+g_{y}\left[m_{I I} Q_{4} \sin Q_{3}+m_{I I} Q_{2}\right]+0.5 \sum_{i=1}^{n s} k_{i}\left\{\left[\left(\cos Q_{3}-1\right) X_{i}-\sin Q_{3} \cdot Y_{i}+Q_{1}\right] .\right. \\
\left.\cdot\left(\cos \alpha_{i}\right)+\left[\sin Q_{3} X_{i}+\left(\cos Q_{3}-1\right) Y_{i}+Q_{2}\right]\left(\sin \alpha_{i}\right)\right\}^{2}+0.5 k_{4}\left[Q_{4}+\bar{x} Q_{3} \sin Q_{3}-\bar{y} Q_{3} \cos Q_{3}\right]^{2}
\end{gathered}
$$

\subsection{Dissipation function of the overall dynamic system}

The dissipation energy $D$ can be introduced in the equations of motion assuming that it is a pseudo-potential energy and is given by the sum of the energy dissipated by the $n d$ dampers characterised by a damping coefficients $d_{\mathrm{j}}$ (whose slopes could be general, different from each other and represented by the angle $\alpha_{\mathrm{j}}$ ) and the dissipation function related to the servohydraulic actuator (whose damping coefficient is name $\mathrm{d}_{4}$ ):

$$
\begin{gathered}
D_{j_{-} \text {dampers }}=0.5 \sum_{j=1}^{n d} d_{j} \bar{v}_{j}^{2}=0.5 \sum_{j=1}^{n d} d_{j}\left[\left(-\dot{Q}_{3} y_{j}+\dot{Q}_{1}\right) \cos \alpha_{j}+\left(\dot{Q}_{3} x_{j}+\dot{Q}_{2}\right) \sin \alpha_{j}\right]^{2} \\
D_{d 4}=0.5 d_{4}\left(\dot{U}_{2}-\dot{U}_{1}\right)^{2}=0.5 d_{4}\left[\dot{Q}_{4}+\bar{x} \dot{Q}_{3}\left(\sin Q_{3}+Q_{3} \cos Q_{3}\right)-\bar{y} \dot{Q}_{3}\left(\cos Q_{3}-Q_{3} \sin Q_{3}\right)\right]^{2}
\end{gathered}
$$

Therefore, the total dissipation function of the dynamic system is calculated as follows:

$$
\begin{aligned}
D & =0.5 \sum_{j=1}^{n d} d_{j}\left[\left(-\dot{Q}_{3} y_{j}+\dot{Q}_{1}\right) \cos \alpha_{j}+\left(\dot{Q}_{3} x_{j}+\dot{Q}_{2}\right) \sin \alpha_{j}\right]^{2}+ \\
& +0.5 d_{4}\left[\dot{Q}_{4}+\bar{x} \dot{Q}_{3}\left(\sin Q_{3}+Q_{3} \cos Q_{3}\right)-\bar{y} \dot{Q}_{3}\left(\cos Q_{3}-Q_{3} \sin Q_{3}\right)\right]^{2}
\end{aligned}
$$




\subsection{Generalised forces applied to the overall dynamic system}

The applied forces $Q_{i}^{*}(\mathrm{i}=1,4)$ are derived from the internal force given by the servohydraulic actuator; therefore, it is in the direction of the degree of freedom $\mathrm{Q}_{4}$. This load could be defined as "follower load" since it is applied in a direction tangential to the centre line of the actuator following the motion of the entire system as it deforms. Being a follower load, the latter cannot be derived from a potential energy. Considering as input signal an external acceleration $\ddot{u}$, the Lagrangian force $Q_{4}^{*}$ applied to system II is given by its mass times the applied acceleration. All the components related to the 4 DOFs are computed as:

$$
Q_{1}^{*}=-\left|m_{I I} \ddot{u}\right| \cos Q_{3}, \quad Q_{2}^{*}=\left|m_{I I} \ddot{u}\right| \sin Q_{3}, \quad Q_{3}^{*}=-\left|m_{I I} \ddot{u}\right| \cos Q_{3} \cdot l, \quad Q_{4}^{*}=\left|m_{I I} \ddot{u}\right|
$$

where $l$ is the distance between the load application line and the centre of mass of system $I$.

\subsection{Derived nonlinear differential equations of motion}

The equations of motion are derived as second-order Lagrange equations:

$$
\frac{d}{d t}\left(\frac{\partial L}{\partial \dot{Q}_{i}}\right)-\left(\frac{\partial L}{\partial Q_{i}}\right)+\left(\frac{\partial D}{\partial \dot{Q}_{i}}\right)=Q_{i}^{*} \quad \text { for } i=1,4
$$

where $L$ is computed as the difference between the total kinetic energy in Eq. (7) and the total potential energy in Eq. (12), $D$ is given in Eq. (15) and the generalised forces in Eq. (16). Writing Eq. (17) with respect to the considered generalised coordinates, four nonlinear differential equations are obtained. For sake of brevity, the equations are not derived herein and the reader may refer to [4] where all the computations are shown and discussed in detail. It has to be pointed out that the strength of the Lagrange's approach is the possibility to directly account for the initial conditions, such as (if any) an initial rotation of the overall dynamic system. It is worth noting that the distribution and the location (Figure 1) of the $k_{\mathrm{i}}$ springs $(i=1$, $n s)$ and the $d \mathrm{j}$ dampers $(j=1, n d)$ are explicitly taken into account in the computations since this is one of the main features of the proposed dynamic model.

\section{SOLUTION OF THE DEVELOPED DETERMINISTIC MODEL}

As already stated in Section 1, one of the aims of this research effort is the formulation of a mathematical model capable of describing the behaviour of a large dynamic testing facility such as the uniaxial shaking table in Figure 1. The Laplace transforms cannot be applied to compute the solutions of the equations of motion since they are nonlinear. Solving these equations of motion accounting directly of the nonlinear contributions is the main feature of the proposed research. Therefore, numerical solution methods capable of solving nonlinear differential equations have been selected. In particular, the fourth order Runge-Kutta method (RK4) is employed because of its good efficiency well documented in literature. However, before solving the equations, preliminary verifications are performed and discussed in the two following sections. The first verification refers to the derivation of simplified models (i.e., "linearised" models), which are well-known in literature. The second verification is related to the validation of the accuracy of the equations and numerical solver. The effects of both time step selection and initial conditions are presented. 


\subsection{Linearised simplified models}

A proof of the accuracy of a developed model for describing very complex systems is the derivation of well-known and simple results. Therefore, some derived simplified models are presented.

a) System with three DOFs $-\mathrm{Q}_{1}, \mathrm{Q}_{2}$ and $\mathrm{Q}_{4}$.

The set of equations describing this system is obtained from a linearization of Eq. (17) neglecting the strain energy $V$, the dissipation function $D$ and the rotation of the system $-V=0$, $D=0, Q_{3}=0$ and $L=T$. The outcome of these assumptions leads to a system whose equations of motion with respect to the coordinates $\mathrm{Q}_{1}, \mathrm{Q}_{2}$ and $\mathrm{Q}_{4}$ are given in Eq. (18). After substituting the third equation in the first one, the two expressions of Eq. (19) are derived. The latter represent the equations of motion of the systems $I$ and $I I$ if they were totally free after the application of the horizontal forces $F_{1 \mathrm{x}}$ and $F_{2 \mathrm{x}}$, acting in opposite directions. Each body develops an inertial force equal to the product of its own mass times its acceleration, in two opposite directions, respectively.

$$
\begin{gathered}
\left\{\begin{array}{l}
\left(m_{I}+m_{I I}\right) \ddot{Q}_{1}+m_{I I} \ddot{Q}_{4}=-F_{1 x}+F_{2 x} \\
\left(m_{I}+m_{I I}\right) \ddot{Q}_{2}=-F_{1 y}+F_{2 y} \\
m_{I I} \ddot{Q}_{1}+m_{I I} \ddot{Q}_{4}=F_{2 x}
\end{array}\right. \\
\left\{\begin{array}{l}
m_{I} \ddot{Q}_{1}=-F_{1 x} \\
m_{I I}\left(\ddot{Q}_{1}+\ddot{Q}_{4}\right)=F_{2 x}
\end{array}\right.
\end{gathered}
$$

b) System with three DOFs $-\mathrm{Q}_{1}, \mathrm{Q}_{2}$ and $\mathrm{Q}_{3}$.

Assuming that the coordinate $Q_{4}$ and the mass $m_{I I}$ can be neglected, the springs and the dashpots are lumped in the centre of gravity of system $I$ and the rotation $\mathrm{Q}_{3}$ is small (i.e., $\mathrm{Q}_{3} \approx$ $0, \sin \mathrm{Q}_{3} \approx \mathrm{Q}_{3}$ and $\cos \mathrm{Q}_{3} \approx 1$ ), the following set of equations is obtained from Eq. (17):

$$
\left\{\begin{array}{l}
m_{I} \ddot{Q}_{1}+c_{11} \dot{Q}_{1}+c_{13} \dot{Q}_{3}+k_{11} Q_{1}+k_{13} Q_{3}=F_{Q 1} \\
m_{I} \ddot{Q}_{2}+c_{22} \dot{Q}_{2}+k_{22} Q_{2}+g_{y} m_{I}=F_{Q 2} \\
\left(I_{x x, I}+I_{y y, I}\right) \ddot{Q}_{3}+c_{33} \dot{Q}_{3}+c_{31} \dot{Q}_{1}+k_{33} Q_{3}+k_{31} Q_{1}=M_{Q 3}
\end{array}\right.
$$

where the $k_{\mathrm{ij}}$ and $c_{\mathrm{ij}}$ coefficients represent the stiffness and the damping of the soils. The derived set of equations is similar to the one originally used in the design phase of an existing shaking table system [1]. The latter was developed for estimating acceleration, velocity and displacement of the reaction mass and studying the soil-structure dynamic interaction. From Eq. (20), it is possible to realise that the vertical motion $Q_{2}$ has no interaction with the horizontal and rotational coordinates, $Q_{1}$ and $Q_{3}$, respectively. This justified the choice of neglecting the vertical degree of freedom during the design phase of the shaking table presented in [1].

\subsection{Accuracy of both derived equations and numerical solver}

The verification of the accuracy of the derived equations is firstly carried out applying the mathematical procedure schematically depicted in Figure 2. Well-known solutions are imposed to the equations of motion and the forces satisfying the equilibrium are computed. The 
latter become the external loads to be applied to the system of equations and the corresponding solutions are calculated. If the latter are similar, within some tolerance error, to the initially imposed solutions, this represents a proof that the equations of motion are accurate and that the numerical solver works properly. Several numerical examples have been studied and a very good agreement between the imposed and computed solutions was always found. In particular, the high quality of the results was due to the application of ad-hoc error tolerance properties: a relative error tolerance is applied to all the components of the solution vector, as well as the absolute error tolerance [6].

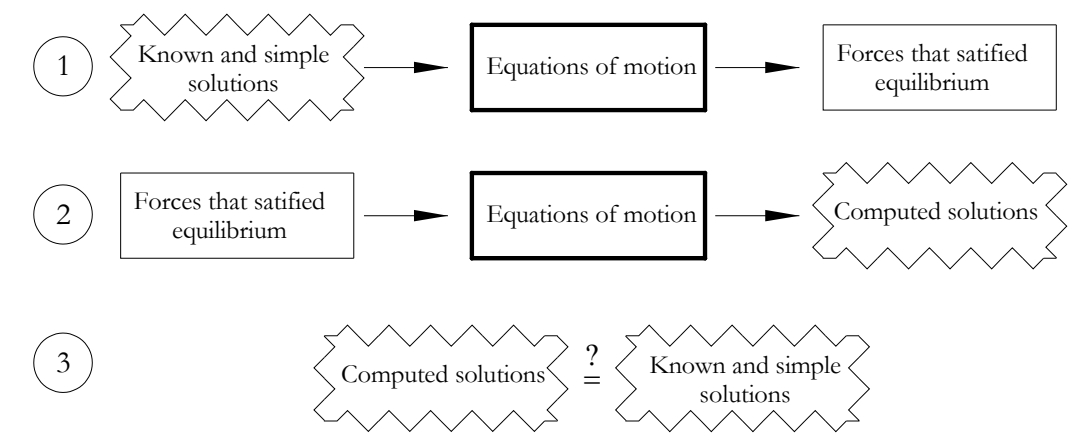

Figure 2: Steps for verifying the accuracy of the derived equations of motion.

Regardless of the integration technique used, the time step remains an important determinant of when to compute the solutions and when to compare the model to data. Fourth order Runge-Kutta integration has an error that is proportional to time step to the fifth power for an integration step and proportional to time step to the fourth power for the whole simulation.

Considering the configuration in Figure 3 with three $\mathrm{DOF}_{\mathrm{s}}-\mathrm{Q}_{1}, \mathrm{Q}_{3}$ and $\mathrm{Q}_{4}$ - and lumped springs $-\mathrm{k}_{1}, \mathrm{k}_{3}$ and $\mathrm{k}_{4}-$ whose nonlinear differential equations are derived from Eq. (17), some verifications of the accuracy of the solutions have been carried out investigating the influence of time step on the numerical solutions. Simple external loads, such as harmonic forces $-\mathrm{F}_{\mathrm{Q} 1}=\mathrm{F}_{\mathrm{Q} 1 \mathrm{o}} \cos (\omega \mathrm{t}), \mathrm{M}_{\mathrm{Q} 3}=\mathrm{M}_{\mathrm{Q} 3 \mathrm{o}} \cos (\omega \mathrm{t}), \mathrm{F}_{\mathrm{Q} 4}=\mathrm{F}_{\mathrm{Q} 4 \mathrm{o}} \cos (\omega \mathrm{t})$ - are applied to the system whose properties are summarised in the Table 1.

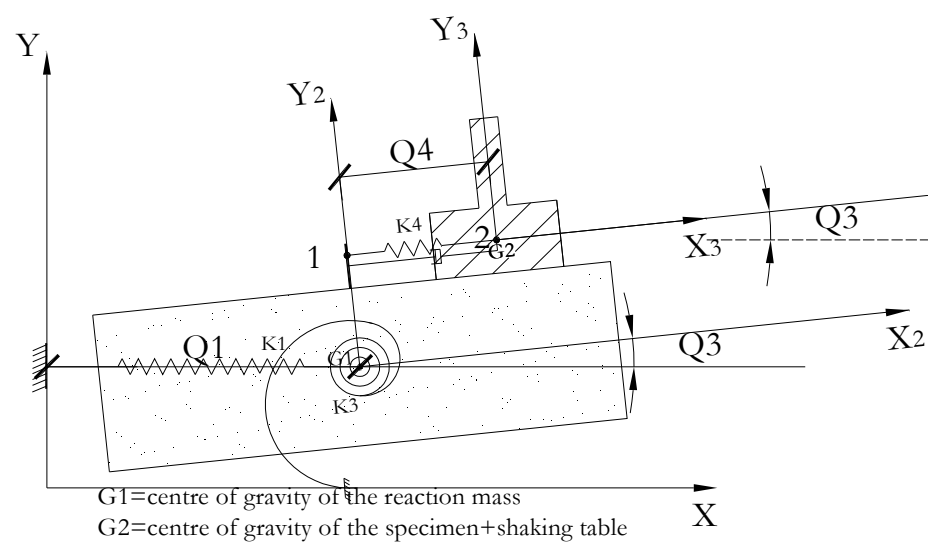

Figure 3: System with 3 generalised coordinates and lumped springs $-\mathrm{Q}_{1}, \mathrm{Q}_{3}, \mathrm{Q}_{4}$ and $\mathrm{k}_{1}, \mathrm{k}_{3}, \mathrm{k}_{4}$, respectively

The corresponding solutions are computed with Runge-Kutta methods using the time step as parameter. Figures 4-6 show the results obtained with a time interval $\Delta t=0.1,0.001$ and 0.00001 seconds, respectively. Unless the first set of solutions in Figure 4, the other two sets 
of results show the same level of accuracy, even if the last one (Figure 6) is much more time consuming.

\begin{tabular}{lllllll}
\hline $\mathrm{k}_{1}[\mathrm{~N} / \mathrm{m}]$ & $\mathrm{k}_{4}[\mathrm{~N} / \mathrm{m}]$ & $\mathrm{k}_{3}[\mathrm{~N} / \mathrm{rad}]$ & $\mathrm{m}_{\mathrm{I}}[\mathrm{kg}]$ & $\mathrm{m}_{\mathrm{II}}[\mathrm{kg}]$ & $\mathrm{I}_{\mathrm{zz}, \mathrm{I}}\left[\right.$ tonnes $\left.\mathrm{m}^{2}\right]$ & $\mathrm{I}_{\mathrm{zz}, \mathrm{II}}$ [tonnes $\left.\mathrm{m}^{2}\right]$ \\
\hline $5 . \mathrm{E}+3$ & $2 . \mathrm{E}+3$ & $10 . \mathrm{E}+3$ & 500 & 5 & 5000 & 100 \\
\hline
\end{tabular}

Table 1: Data of the numerical example for proving the effect of time step choice on the solutions of the system
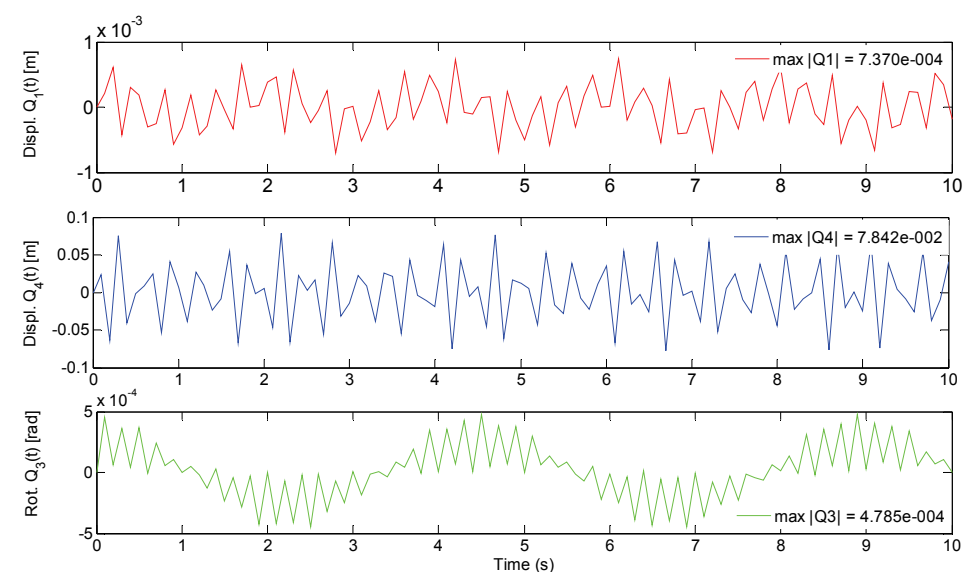

Figure 4: Numerical solutions of the system in Figure 3 for $\Delta t=0.1 \mathrm{~s}$ with Runge-Kutta (RK4) method
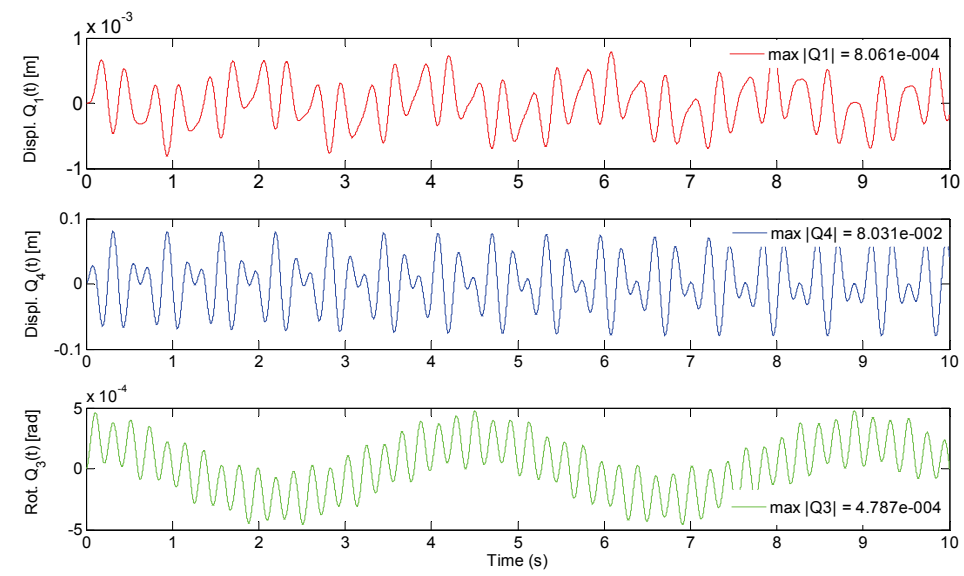

Figure 5: Numerical solutions of the system in Figure 3 for $\Delta t=0.001 \mathrm{~s}$ with Runge-Kutta (RK4) method
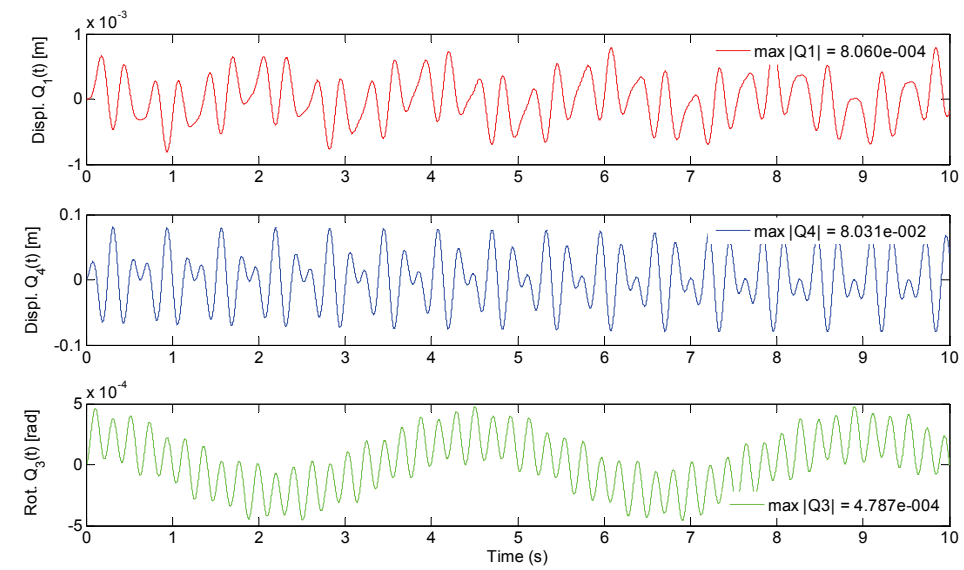

Figure 6: Numerical solutions of the system in Figure 3 for $\Delta t=0.00001 \mathrm{~s}$ with Runge-Kutta (RK4) method 
As previously stated, one of the strengths of the Lagrange's approach is the possibility of taking into account the initial conditions. The influence of the initial conditions on the computed solutions is evaluated considering the system in Figure 3 subjected to an initial rotation $\mathrm{Q}_{30}=-0.01 \mathrm{rad}$. The numerical responses due to the application of the harmonic forces $\mathrm{F}_{\mathrm{Q} 1}$, $\mathrm{M}_{\mathrm{Q} 3}$ and $\mathrm{F}_{\mathrm{Q} 4}$ (previously defined) change in a non-negligible manner as it can be evaluated comparing the new time-histories plotted in Figure 7 with the ones in Figure 5. Since the damping has been neglected in this numerical case-of-study, the effect of the imposed initial rotation continues endlessly. This is only a simple example showing the interaction between the degrees of freedom and the influence of the initial rotation on the translational DOFs, $Q_{1}$ and $\mathrm{Q}_{4}$. However, the damping is present in the real system and it makes the free vibration decay with time. One important outcome of this numerical case-of-study is that, if any initial rotation affects the overall dynamic system, its effect strongly influences the displacements, velocities and accelerations of the entire dynamic facility. Then, the transient effect disappears due to the effect of damping coefficients [5].

It can be stated that the contributions of the initial conditions (if any) should be accounted for during the experimental tests. One of the main advantages of the derived model is that the initial conditions can be directly considered and the computed responses could be used as predictions of the behaviour of the dynamic facility.
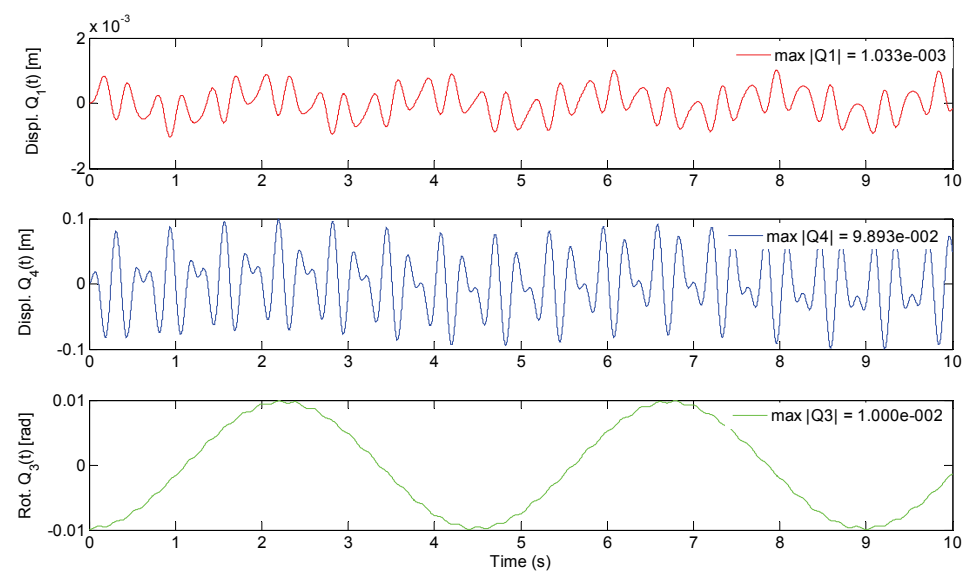

Figure 7: Numerical solutions of the system in Figure 3 for $\Delta t=0.001 \mathrm{~s}$ with Runge-Kutta (RK4) method and $\mathrm{Q}_{30}=-0.01 \mathrm{rad}$

\section{MODELLING THE RESPONSE OF AN EXISTING LARGE-SCALE DYNAMIC TESTING FACILITY}

The developed model was formulated in a general manner. Therefore, it works or it could be validated considering any type of uniaxial shaking table system. In order to evaluate the accuracy of the equations of motion, numerical verifications should always be carried out, whenever feasible. The case-of-study for verifying the nonlinear differential equations is the large uniaxial shaking table built in the Laboratory of Eucentre (European Centre for Training and Research in Earthquake Engineering, www.eucentre.it), Pavia (Italy). The latter is chosen for its large dimensions and powerful performances in terms of stroke, velocity and accelerations applicable to the payload (Table 2). Furthermore, the system composed by the reaction mass, the shaking table and the specimen constitutes a non-equilibrated structure due to the eccentric position of both the platform and the specimen to be tested. The former is eccentric with respect to the centre of gravity of the reaction mass, the latter for its own configuration. Therefore, the system could suffer rotation and be characterised by a nonlinearity of the response. Moreover, it has been measured that the reaction mass suffered a rigid rotation proba- 
bly due to the settlement of the system [7]. This rotation could be seen as an initial condition to be taken into account in predicting the response of such a complex dynamic testing facility.

Figure 8 shows the adopted solution for the system restraining the motion of the platform using hydrostatic bearings at each side and below the table in order to avoid transversal and vertical movements (all the details can be found in [1]).

The available data belong to the experimental campaign performed during the calibration phase of the dynamic facility [8] for the evaluation of the dynamic response of soil under the vibrations produced by the shaking table. Some of these data have been discussed in [4] in order to prove the rocking affecting the motion of the reaction mass. Additional comparisons are presented in the following sections.

\begin{tabular}{ll}
\hline Main properties & \\
\hline Table size & $5.6 \mathrm{~m} \times 7.0 \mathrm{~m}$ \\
Table mass & 35 tonnes \\
Maximum specimen mass & 60 tonnes \\
Controlled degree of freedom & $\mathrm{X}$ (longitudinal) \\
Peak displacement & $\pm 500 \mathrm{~mm}$ \\
Peak velocity & $\pm 1500 \mathrm{~mm} / \mathrm{s}$ \\
Peak acceleration (bare table condition) & $\pm 6.0 \mathrm{~g}$ \\
Maximum static force & $2100 \mathrm{kN}$ \\
Maximum dynamic force & $1700 \mathrm{kN}$ \\
Maximum overturning moment capacity & $4000 \mathrm{kNm}(1000 \mathrm{kN}$ at $4 \mathrm{~m}$ \\
& from the top plate of the platform) \\
Maximum yaw moment & $400 \mathrm{kNm}$ \\
Operating frequency range & $0-50 \mathrm{~Hz}$ \\
\hline
\end{tabular}

Table 2: Performance characteristics of the Eucentre shaking table [1]

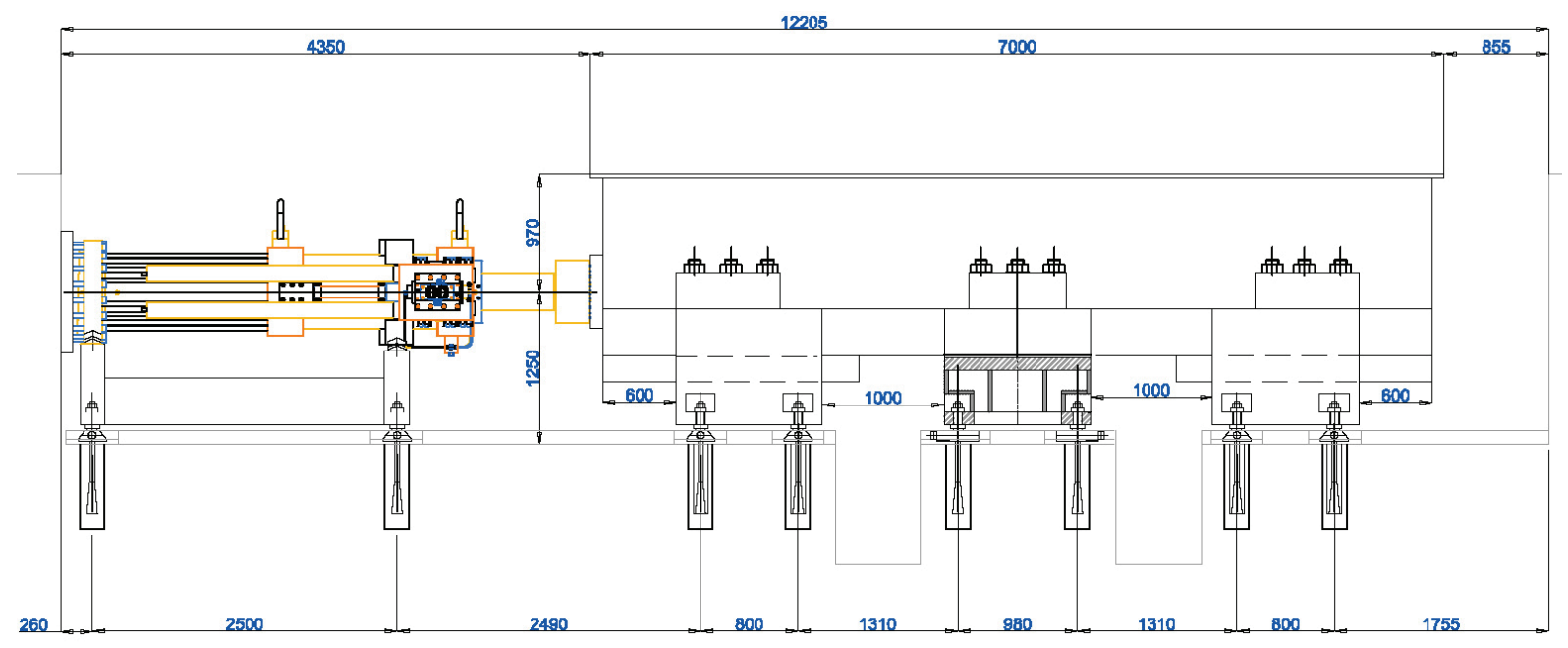

Figure 8: Longitudinal view of the final hydrostatic and mechanical configuration of the shaking table

\subsection{First available experimental case-of-study}

This test was characterised by the sudden stop while retracting the actuator and then consequent free vibration response. The objectives of the test were the analysis of the actual motion of the reaction mass and the fundamental frequency of the response under the excitation provided. Five servo-accelerometers were located over the reaction mass and one over a col- 
umn of the laboratory (Figure 9). The test was performed at low-magnitude and excitation and bare-table condition. During this test, the rocking of the reaction mass was measured as shown in Figure 10. The observed response is complex and suggests that the distribution of the stiffness and damping coefficients of the soil is not uniform in the longitudinal direction, which is the direction of both the application of the force and the allowed motion of the dynamic testing facility.

The first application of the developed model attempts to reproduce the observed experimental results taking into account that the record of the excitation applied to the shaking table during the sudden stop while retracting the actuator is not available. Some assumptions were introduced for applying a reasonable forcing function and finding the solutions. A rectangular pulse was applied whose amplitude was related to the quantity of motion of the dynamic facility, i.e., the mass of the shaking table and its velocity. Some configurations of springs and dashpots were studied in order to optimise the simulation of the measured accelerations at both sides of the reaction mass, and in the horizontal and vertical directions. Due to the coupling of the degrees of freedom and the presence of nonlinear terms in the equations of motion (Section 2), the calibration procedure was not straightforward. The computed responses are plotted in Figure 11. The ratios between the maximum absolute amplitudes of accelerations are compared in Table 3. Taking into account the several unknowns of the case-study (i.e., the time-history, amplitude and duration of the applied force, and the right distribution of stiffness and damping coefficients of the soil), the obtained solutions are quite satisfactory and the rocking of the reaction mass is reproduced.

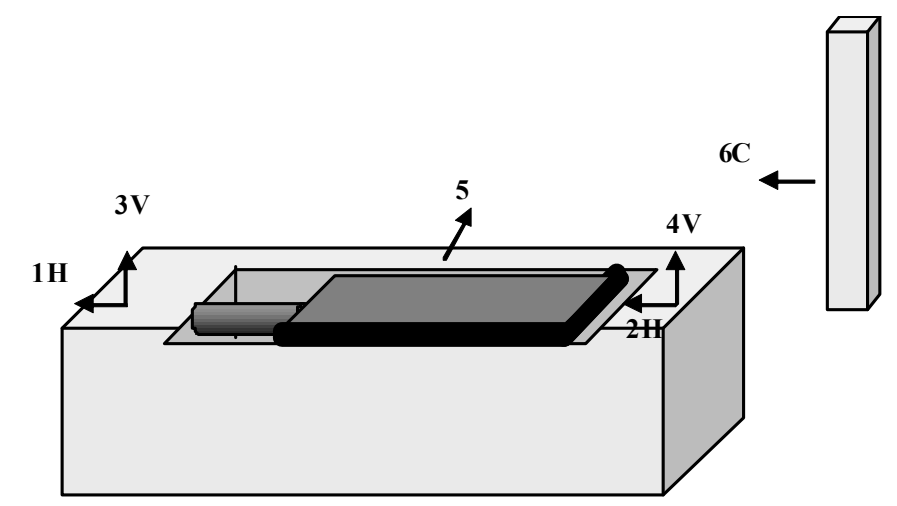

Figure 9: Location of the instrumentation for the first case-of-study
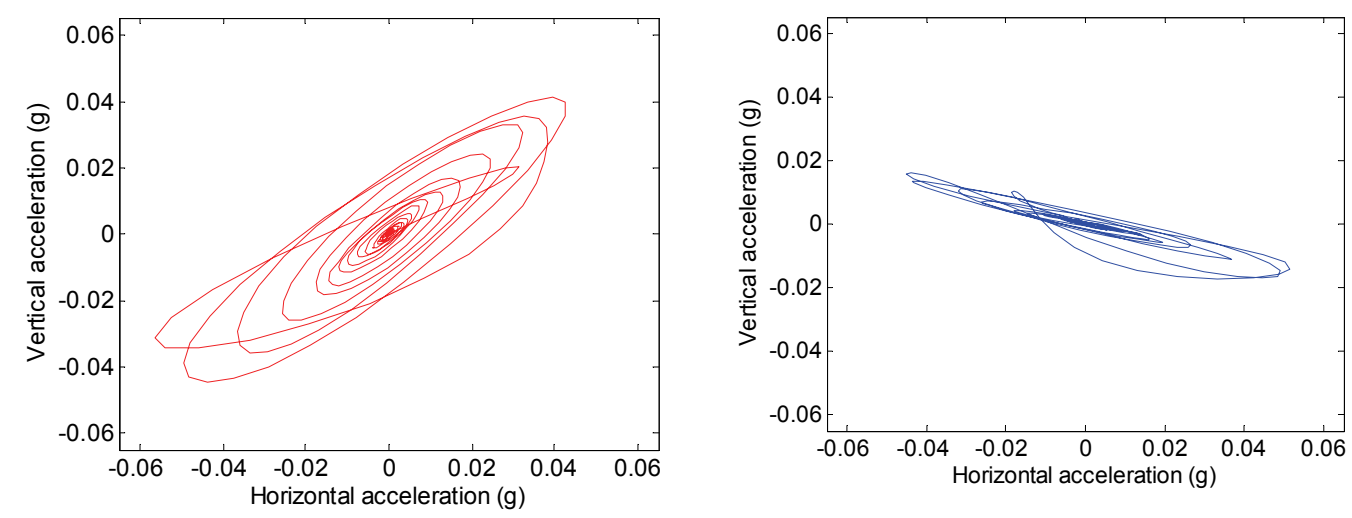

Figure 10: Compositions of the measured acceleration components at the two sides of the reaction mass $-1 \mathrm{H}$ vs $3 \mathrm{~V}$ (at the actuator side) on the left, $2 \mathrm{H}$ vs $4 \mathrm{~V}$ (at the opposite side) on the right 

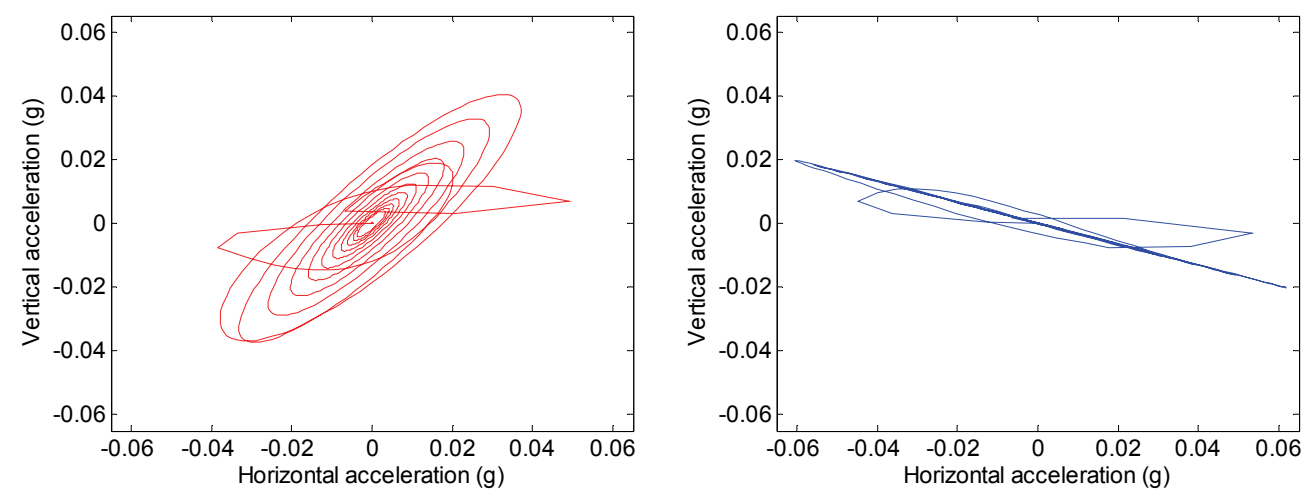

Figure 11: Compositions of the computed acceleration components at the two sides of the reaction mass $-1 \mathrm{H}$ vs $3 \mathrm{~V}$ (at the actuator side) on the left, $2 \mathrm{H}$ vs $4 \mathrm{~V}$ (at the opposite side) on the right

\begin{tabular}{ll}
\hline \multicolumn{2}{l}{ Measured accelerations } \\
\hline $1 \mathrm{H} / 2 \mathrm{H}$ & 1.091 \\
$3 \mathrm{~V} / 4 \mathrm{~V}$ & 2.576 \\
$1 \mathrm{H} / 3 \mathrm{~V}$ & 1.263 \\
$2 \mathrm{H} / 4 \mathrm{~V}$ & 2.984 \\
\hline Computed accelerations & \\
\hline $1 \mathrm{H} / 2 \mathrm{H}$ & 0.789 \\
$3 \mathrm{~V} / 4 \mathrm{~V}$ & 2.003 \\
$1 \mathrm{H} / 3 \mathrm{~V}$ & 1.209 \\
$2 \mathrm{H} / 4 \mathrm{~V}$ & 3.067 \\
\hline
\end{tabular}

Table 3: Ratios between the maximum absolute amplitudes of accelerations of the first case-of-study

\subsection{Second available experimental case-of-study}

The input motion used during this test was the Coalinga record at $200 \%$ magnitude. During this test a rigid payload of 60 tonnes composed by a layer of reinforced concrete cubic elements was located on the shaking table. The location of the instrumentation is shown in Figure 12: a geophone was located on the centre right hand side of the reaction mass plus eight servo-accelerometers located at the borders and edges of the reaction block. The available experimental data are the accelerations acquired by the servo-accelerometers $(1 \mathrm{~V}-8 \mathrm{H}$ in Figure 12). Figure 13 presents the time-histories of the vertical and horizontal components in three main locations of the reaction block. The label "down" refers to the signals measured at location C (Figure 12), "up-actuator" at the location B and "up-opposed" at location A. The figures show that there is a large discrepancy in the attenuation of the signals at the two sides of the reaction mass, reaching lower values at the side opposite to the actuator anchorage location - "up-actuator" vs "up-opposed". Very different results are obtained even at the same side of the reaction block - "down" vs "up-actuator". These measured response show that the behaviour of the Eucentre shaking table is complex.

The input signal applied during this test is not available. Therefore, the Coalinga record considered during the numerical simulation has been downloaded from the PEER strong motion database (http://peer.berkeley.edu/smcat) and then doubled. For this reason, the comparison between the measured and computed results is performed in terms of ratios between the peak response quantities. Table 4 compares the peak values of the numerical solutions versus 
the measured ones. Notwithstanding with the unavailability of the real applied input signal, the obtained solutions are in good correlation with the measured peak responses. The amplitudes of the computed solutions strongly depend on the assigned distribution of the coefficients introduced for modelling the stiffness and the damping of the soil. The latter have to be deeply investigated.

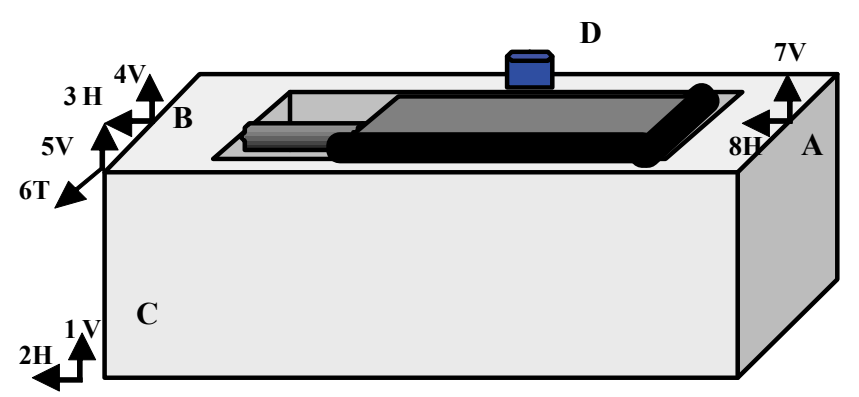

Figure 12: Location of the instrumentation for the second case-of-study
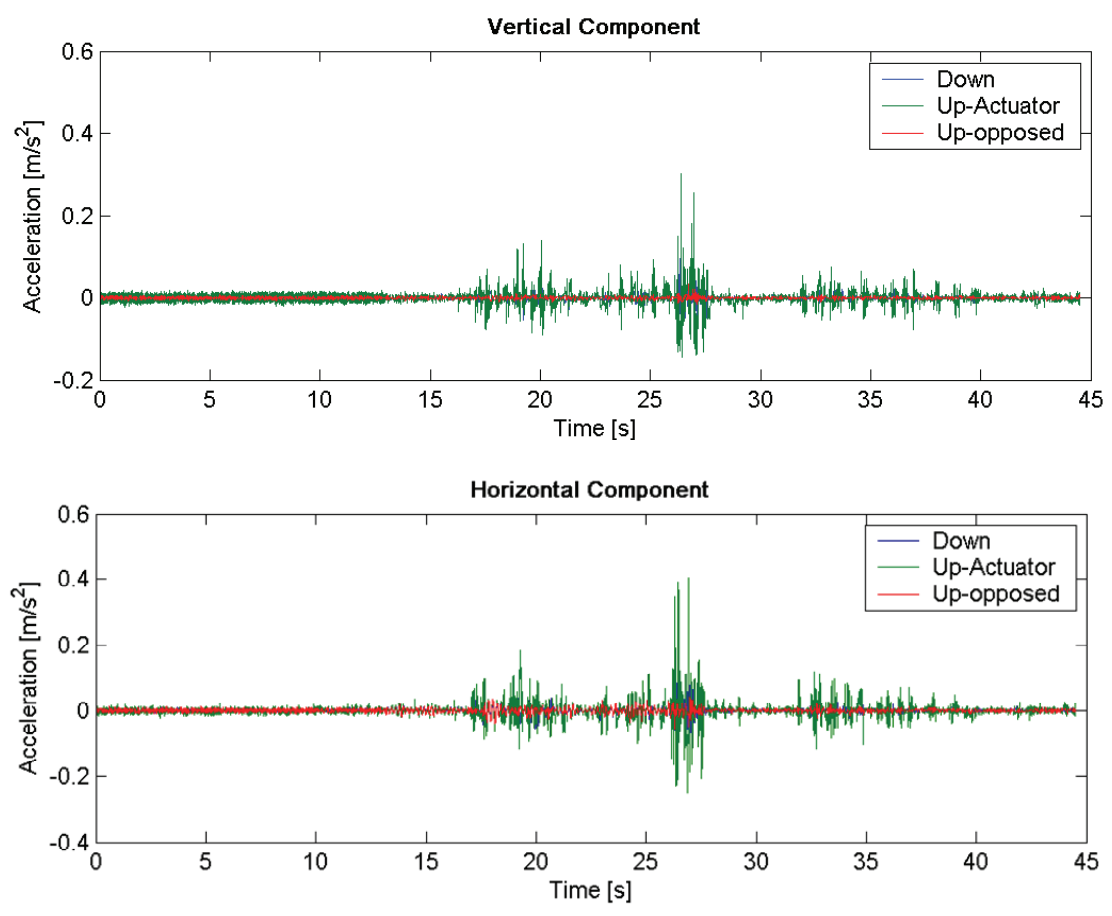

Figure 13: Vertical and horizontal accelerations recorded by the servo-accelerometers in 3 main locations on the reaction mass. With reference to Figure 12, label "Down" refers to location C, "Up-Actuator" to B, "Upopposed" to A

\begin{tabular}{lll}
\hline Acceleration components & Peak values & Ratio Num./Exp. \\
\hline Horizontal & Positive & 0.95 \\
& Negative & 1.10 \\
Vertical & Positive & 1.18 \\
& Negative & 0.93 \\
\hline
\end{tabular}

Table 4: Comparison of the peak accelerations of the second case-of-study: solutions of the proposed model vs acquired experimental responses (in Figure 13) at location B (shown in Figure 12) 


\section{CLOSURE}

As stated by Crewe and Severn [9], for seismic testing the most used input is that of translation in one, or sometimes two or three directions, with rotations generally being small and unwanted. Following this statement, the proposed model has been formulated for simulating the performance of a shaking table whose longitudinal motion is produced by a servohydraulic actuator oriented in the horizontal direction. However, the nonlinear kinematics due to the possible rocking of the support system (i.e. reaction-mass/isolation-dampingsystems/foundation-soil) are directly considered in the formulation since a large rotation associated with an intended uniaxial input could have a very strong influence on the response of the shaking table and the specimen. The novelty of the proposed research stays in the explicit consideration of the effects of nonlinear kinematics with the application of the motion composition rules (the velocity composition rule and Coriolis' theorem).

The model has been used to evaluate "a posteriori" if the performance of an existing shaking table could be affected by large rotations. The EUCENTRE shaking table has been considered. It is worth pointing out that the collected tests do not represent an exhaustive set of examples for validating the model and additional experimental data are required for calibrating the analytical model and characterising the motion of the dynamic facility. Notwithstanding the few available experimental data, the preliminary numerical validations seem very promising encouraging further developments and applications.

Further improvements of the results should be reached if rigorous optimisation methods will be implemented. One such is least-squares identification [10], in which a numerical optimisation routine selects the best combination of parameters so as to minimise the fit error.

\section{ACKNOLEDGMENTS}

The authors thank Mr. J. Londono Bustamante, Mr. F. Dacarro, Mr. R. Franzolin, Prof. A. Pavese and Dr. C. Strobbia for the assistance in retrieving experimental data related to the case-study considered in this research work. Many thanks are given to Dr. R. Pinho for his constant support throughout the progress of this research work.

\section{REFERENCES}

[1] G.M. Calvi, A. Pavese, P. Ceresa, F. Dacarro, C.G. Lai, C. Beltrami, Design of a LargeScale Dynamic and Pseudo-Dynamic Testing Facility. IUSS Press, 2005.

[2] G. Legnani, P. Righettini, B. Zappa, F. Casolo, A Homogeneous Matrix approach to 3D Kinematics and Dynamics. Part 1: Theory. Mechanics and Machine Theory, 31(5), 573587, 1996.

[3] G. Legnani, Robotica Industriale. (in Italian) Casa Ed. Ambrosiana 2003.

[4] P. Ceresa, F. Brezzi, G. M. Calvi, R. Pinho, Analytical modelling of a large dynamic testing facility. Earthquake Engineering and Structural Dynamics, 41, 255-277, 2012.

[5] A. K. Chopra, Dynamics of structures. Second Edition, Prentice Hall, 2001.

[6] The MathWorks, Inc. Matlab, The Language of Technical Computing, 2002. 
[7] DIET, Misure delle variazioni di quota dei punti di controllo situati sul basamento della tavola vibrante (in Italian), Report of the Dipartimento di Ingegneria Edile e del Territorio, University of Pavia, 2007.

[8] J. Londono, Dynamic response of soil under vibrations produced by the Eucentre shaking table, Master Thesis (in preparation), ROSE School, Pavia, Italy.

[9] A.J. Crewe, R.T. Severn, The European Collaborative programme on evaluating the performance of shaking tables, Philosophical Transactions of the Royal Society A: Mathematical, Physical \& Engineering Sciences, 359(1786), 1671-1696, 2001.

[10] G.F. Franklin, J.D. Powell, A. Emami-Naeini, Feedback control of Dynamic Systems, Third Edition, Addison-Welsey Publishing Company, 1994. 